\title{
ASSOCIAÇÃO IMOBILIDADE NA INTERNAÇÃO HOSPITALAR DE IDOSOS E MORTE: ESTUDO COM 3 ANOS DE SEGUIMENTO
}

Liege Camargo Alves Kurrle; Laboratório de Biogenômica, Universidade Federal de Santa Maria; liegekurrle@gmail.com

Fernanda Barbisan; PPG Gerontologia, Universidade Federal de Santa Maria; fernandabarbisan@gmail.com

Thamara Graziela Flores; PPG Farmacologia, Universidade Federal de Santa Maria; thamaraflores_fisio@yahoo.com.br

Neida Luiza K. Pellenz; Departamento de Enfermagem, Universidade Federal de Santa Maria; neidaluiza@gmail.com

Ivana Beatrice Mânica da Cruz; PPG Gerontologia, Universidade Federal de Santa Maria; ibmcruz@hotmail.com

Melissa Agostini Lampert; PPG Gerontologia, Universidade Federal de Santa Maria; melissa.a.lampert@gmail.com

\section{RESUMO}

Introdução: A imobilidade durante internações hospitalares pode ter impacto no sistema musculoesquelético favorecendo outras doenças e a síndrome sarcopenica, bem como no sistema emocional dos idosos. Objetivo: Analisar a associação entre imobilidade intra-hospitalar e mortalidade em idosos após 3 anos de seguimento. Método: Trata-se de um estudo quantitativo, longitudinal e descritivo. A coleta de dados inicial ocorreu no ano de 2015 - 2016 e o desfecho mortalidade foi analisado nos anos de 2017-2020. A amostra proveniente do Hospital Universitário de Santa Maria, composta inicialmente por 493 idosos, sendo que 165 foram excluídos por não apresentarem 2/3 dos dados necessários para o estudo ou à ausência de contato. Análise estatística via software SPSS versão 21.0, por análise univariada do teste de qui-quadrado ou exato de Fisher e a razão de chances (odds ratio) através da regressão logística (modelo Backword). Valores significantes foram considerados quando $\mathrm{p}$ valor $<0,05$. Resultado: Observou-se 4,6\% $(\mathrm{n}=15)$ apresentavam imobilidade prévia a internação e que 43,0\% $(n=141)$ apresentavam imobilidade de causa hospitalar, destes $53,2 \%(n=75)$ eram do sexo masculino, com idade entre 60 e 69 anos(41,8\%), a maior prevalência de imobilidade (31,9\%) foi devido a fraturas. Observou-se associação entre as variáveis imobilidade de causa hospitalar e óbito no período de 3 anos após a internação( $p=0,039)$. Conclusão: A associação entre imobilidade intra-hospitalar de idosos e mortalidade destes idosos após 3 anos de seguimento foi encontrada. Estes dados reforçam a necessidade de o paciente manter-se ativo, dentro de suas possibilidades, durante o período de internação.

Palavras-chaves: Imobilidade; Idoso; Hospitalização.

Agradecimentos: CAPES, CNPQ, PROIC-HUSM. 